EOMmUn Communication et organisation

Dम매I 32 | 2007

La ville dans tous les sens

\title{
La ville dans tous les sens
}

Les nouvelles écritures de la ville

Nicole Denoit et Marie-Pascale Mignot

\section{(2) OpenEdition}

Journals

Édition électronique

URL : http://journals.openedition.org/communicationorganisation/245

DOI : 10.4000/communicationorganisation.245

ISSN : 1775-3546

Éditeur

Presses universitaires de Bordeaux

Édition imprimée

Date de publication : 1 décembre 2007

Pagination : 8-13

ISSN : 1168-5549

Référence électronique

Nicole Denoit et Marie-Pascale Mignot, "La ville dans tous les sens ", Communication et organisation [En ligne], 32 | 2007, mis en ligne le 01 décembre 2010, consulté le 21 septembre 2020. URL : http:// journals.openedition.org/communicationorganisation/245; DOI : https://doi.org/10.4000/ communicationorganisation.245 
Nicole Denoit est docteur en Littérature Française et en Sciences de l'Information et de la Communication. Elle enseigne les Sciences de l'Information et de la Communication à l'UFR Lettres Langues de l'Université François Rabelais de Tours. Ses recherches portent sur la communication institutionnelle des entreprises, leur implication dans la cité et sur les formes de la médiation culturelle, en particulier dans le tissu urbain.

Marie-Pascale Mignot est architecte. Maître de conférence associée à l'ISIC (Institut des Sciences de l'Information et de la Communication), elle est co-responsable du parcours stratégies et produits de communication du master Communication des organisations de l'ISIC, Université Michel de Montaigne-Bordeaux 3. Ses recherches portent sur la place de l'espace dans les processus de communication. 


\section{Dossier \\ La ville dans tous les sens \\ Les nouvelles écritures de la ville \\ Nicole Denoit et Marie-Pascale Mignot \\ nicole.denoit@univ-tours.fr mignot@u-bordeaux3.fr}

Le dossier de Communication \& Organisation que nous avons intitulé « la ville dans tous les sens » s'intéresse à un espace urbain dont nous avons voulu saisir à la fois l'importance (plus de $80 \%$ de la population française vit en ville; c'est aussi vrai ailleurs), la mutation (métropolisation, globalisation) et la complexité, véritable " polyphonie urbaine » comme l'écrit Bernard Lamizet à propos de la communication urbaine dont le propos ouvre ce dossier. Sous l'angle de la communication, nous avons souhaité que soit posée la question de la limite, à un moment de notre société de nouvelles technologies où les frontières sont invisibles, sans cesse repoussées plus loin, aussi loin que les communications le permettent, apportant un possible infini.

Dans ce contexte, sans vouloir la dramatiser, la ville apparaît aujourd'hui comme un théâtre passionnant d'expressions multiples, métissées, contradictoires, qui interrogent notre société en mutation accélérée. C'est donc une ville multiple, à la fois hétérogène et sectorisée, traversée par des réseaux de fonctionnalité, des réseaux affectifs, habillée par une complexité de messages contradictoires, qui est analysée dans ce dossier consacré aux «nouvelles écritures de la ville ». Bernard Lamizet prend en charge, dans son propos, l'hétérogénéité de la ville pour analyser la polyphonie de la communication dans un espace urbain qui met en scène une confrontation incessante entre des langages et des cultures à travers l'histoire. Il montre que cette diversité, cette hétérogénéité produisent une polyphonie et non une cacophonie car il y a bien une logique de médiation esthétique qui organise la multiplicité des codes de la ville. Il souligne que la "polyphonie urbaine » est une médiation en ce qu'elle institue une dialectique entre le singulier et le collectif, préservant la spécificité de chaque élément tout en l'intégrant à un système collectif. Ce mode d'articulation du singulier au collectif renvoie à une polyphonie visible des paysages urbains, une polyphonie audible, une polyphonie lisible. Or, l'intitulé de ce dossier «La ville dans tous les sens", montre que l'aspect sensoriel nous intéresse au plus haut point. 
C'est autour de ces approches sensorielles, sensibles, que se rencontrent les recherches d'Emmanuelle Lallement, Talya Bigio et Patrick Barrès, sur les nouvelles scénographies urbaines, événementielles et architecturales, que permettent les nouvelles techniques, qu'elles soient informatiques, numériques ou médiatiques. La ville se resserre autour d'images façonnées, de scènes organisées, comme pour parer à une évasion du territoire. Emmanuelle Lallement, sous le titre "Evénements en ville, événements de ville: vers de nouvelles ritualités urbaines?» se place sur le terrain de ces scénographies urbaines pour en analyser les stratégies, les effets recherchés de leur ritualisation dont elle présente les points communs. L'auteur montre comment "l'événementiel répétitif» fonctionne comme une rupture attendue du cours ordinaire du rythme urbain. Elle souligne le caractère codifié de ce type d'événements, véritable détournement matériel et symbolique opéré sur la ville, reconquête du temps et de l'espace par le biais de ces manifestations festives - Parisplage, Nuits Blanches, Nuit des musées - qui, lorsqu'elles se déroulent la nuit, prennent une dimension transgressive. C'est aussi toute la logique d'une mise en scène de l'insolite urbain qui est analysée. Emmanuelle Lallement, comme Talya Bigio, nous parlent de la manière dont l'individu réagit aujourd'hui dans son corps aux stratégies de la communication urbaine qui, par des propositions artistiques, architecturales, culturelles, transforment le rapport à l'espace urbain et de ce fait à la collectivité, au groupe social, faisant éprouver ainsi, comme le dit Emmanuelle Lallement, le besoin de « se réaffirmer en décidant d'un temps, d'un espace et d'une activité à la fois très codifiée et décalée par rapport à la vie ordinaire ». Talya Bigio interroge l'occupation paradoxale d'un espace public, pourtant sous haute surveillance, par une mise en visibilité de l'intime, dont l'ampleur est rendue possible par les nouvelles technologies de communication. L'auteur annonce également un nouveau rapport au temps, à l'espace, au corps dans son intimité : confusion du dehors et du dedans analysée à travers différentes scénographies urbaines qui font date. C'est une véritable poétique de l'espace qu'analyse l'auteur pour montrer le paradoxe d'un espace urbain qui serait tout à la fois celui d'une société du divertissement et d'une société de surveillance généralisée.

Patrick Barrès désigne par ce qu'il appelle un « espace architectural en pli» les constructions actuelles qui privilégient la fluidité par l'utilisation de matériaux permettant des dynamiques plastiques innovantes "instaurant une tension permanente entre le flux et le lieu », soit « des systèmes de façade-enveloppe, tels les trames, les 
tresses, les alvéoles et les résilles, les motifs de plis-sur-plis (des plis en mouvement) ». Le propos est architectural, esthétique. Cependant il est remarquable de retrouver dans l'analyse du nouveau traitement des matériaux par l'architecte, notamment Franck Gehry et Massimiliano Fuksas, «le fragment, la discontinuité, l'hétérogène (...) des dislocations, des symétries contradictoires" qui ne sont pas sans rappeler l'hétérogénéité des éléments de la "polyphonie urbaine" dont traite Bernard Lamizet; de même cette valorisation expérimentale en architecture d' «une part d'indéterminé, d'improbable, de mobile ou de varié », que présente Patrick Barrès, fait écho à l'aspect hasardeux, aléatoire de la communication urbaine polyphonique.

Bernard Lamizet introduit la «polyphonie urbaine », Emmanuelle Lallement les ritualisations en rupture, en décalage, Talya Bigio le paradoxe d'une interpénétration de l'intime et du public, Patrick Barrès à propos des nouvelles architectures de la ville parle de discontinuité, de dislocations, de ruptures avec les normes instaurées. Jusque dans ses murs la ville est en rupture avec elle-même. Ce que les articles d'Emmanuelle Lallement et de Talya Bigio nous disent du vivre ensemble, Patrick Barrès l'inscrit dans le traitement architectural, esthétique, des matériaux qui édifient la cité d'aujourd'hui.

L'architecture est également au cœur de l'analyse que propose Béatrice Galinon-Mélénec sous le titre «Voiles et dévoilements du Havre ", dont le patrimoine architectural, que l'on doit à Auguste Perret, longtemps le mal-aimé des Havrais, vient d'être classé par l'UNESCO, au Patrimoine de l'Humanité. Par un regard sensible, l'auteur nous fait voir les éblouissements d'une lumière propre à cette ville portuaire qui a fasciné les peintres, nous fait sentir les embruns, les brumes qui voilent cette «métropole maritime» où se croisent des populations de tous milieux, venues de tous les coins du monde dans des perspectives très diverses aussi bien commerciales que touristiques ou même militaires. Le Havre, Ville Port, induit dans l'imaginaire collectif les rêves ou les cauchemars que cette taxinomie engendre. Le Havre, comme ville portuaire, est d'abord un " espace d'émotions ", au travers duquel nous guide Béatrice Galinon Mélénec pour nous faire comprendre, par une analyse propre aux Sciences de l'Information et de la Communication, comment deux organisations, la mairie du Havre et l'UNESCO, ont mis en œuvre des processus de reconstruction de représentation de cette ville. En effet, le Havre est une ville qui mérite qu'on s'y sente bien et pourtant elle ne s'aime guère. Les Havrais se sont repliés sur eux-mêmes depuis les 
destructions de la guerre; ils ont perdu confiance. C'est donc une image négative que la ville donne à lire et dont les médias se font le relais. La réalité urbaine est ici approchée dans la construction et le fonctionnement de ses représentations, pour suivre la mise en œuvre d'une transformation fondamentale qui va s'opérer dans les mentalités, par les stratégies de communication, les médiations culturelles ouvrant à «la poétique du béton » pour que l'identité du Havre soit perçue positivement au niveau local jusqu'à être reconnue au niveau mondial dans l'audace de sa reconstruction architecturale. La perspective urbaine ouverte par Béatrice Galinon-Mélenec est à la fois architecturale et humaine. Elle nous parle du bâti mais aussi de la mémoire et de la sensibilité de ceux qui habitent la ville, de la manière dont ils s'y sentent vivre.

Le lien est donc naturel entre son propos et celui de Ghyslaine Thorion sous le titre « La médiation, moyen de prévenir la crise urbaine en France » qui ouvre une série de trois articles orientés vers la question sociale dans l'approche de la ville. L'auteur, spécialiste de la médiation en Sciences de l'Information et de la Communication, analyse l'enjeu et l'efficacité des actions politiques et sociales mis en œuvre par des programmes successifs de réhabilitation de l'habitat social, d'insertion par l'économique et le désenclavement des zones urbaines sensibles, montrant que «cette politique de rénovation urbaine a permis d'institutionnaliser la médiation comme mode de régulation sociale ». Cependant, si l'importance de la médiation sociale comme initiative citoyenne est reconnue par les pouvoirs publics comme "gage de paix sociale », il convient de comprendre qu'elle ne peut résoudre seule les problèmes de la cité, en particulier de ses franges défavorisées, et que les politiques publiques, loin d'y trouver un alibi à leur déficience, doivent se donner les moyens de répondre aux besoins que fait apparaître, sur le terrain, cette « veille sociale » précieuse réalisée par les médiateurs. Un dossier sous-titré «les nouvelles écritures de la ville» ne pouvait se concevoir sans une réflexion sur les transformations du «vivre ensemble» opéré par le développement des nouvelles techniques de communication.

Margot Beauchamps, sous le titre «Internet : vers une fragmentation de la ville», annonce explicitement la teneur de son analyse: s'appuyant sur différentes enquêtes, notamment celles de l'INSEE, elle montre que les inégalités profondes perceptibles entre les habitants des différents quartiers de la ville correspondent aux inégalités de l'accès des citadins aux TIC. En effet, l'auteur explique que le développement des TIC est un démultiplicateur d'inégalités sociales en milieu urbain, que la fragmentation des quartiers en 
fonction de leur niveau de raccordement aux réseaux met en péril la cohésion urbaine qu'avaient justement forgée les réseaux urbains déployés au $\mathrm{XIX}^{\mathrm{e}}$ et $\mathrm{XX}^{\mathrm{e}}$ siècles (réseaux électriques, réseaux d'assainissement...). Il est donc capital que les pouvoirs publics prennent conscience de la gravité de cette disparité dans l'appropriation des TIC, s'appuient sur les structures administratives et les structures associatives existantes, dotent « les travailleurs sociaux de compétences nouvelles leur permettant d'inclure la dimension TIC dans l'aide qu'ils fournissent aux personnes en difficulté, notamment dans un défaut d'accès aux ressources informationnelles ». La dimension humaine d'une approche de la ville d'aujourd'hui ne pouvait être enfermée dans nos frontières. Il fallait que la question sociale du monde urbain précisément s'ouvre au monde.

Cette ouverture est proposée par Joanne et Paul Rasse sous le titre « La planète Bidonville, le cas des Chabolas de Madrid », croisant les compétences de l'architecte et du chercheur en Sciences de l'Information et de la Communication. L'article se propose de «rendre compte d'une enquête urbanistique menée sur les Chabolas de Madrid, et de la mettre en perspective dans une approche anthropologique des grandes mutations entraînées par l'essor des moyens de communication» conjuguant «l'observation patiente d'une microsociété avec une mise en perspective des connaissances recueillies, en les resituant dans un projet anthropologique global, plus vaste, d'analyse de la civilisation dans ses aspects planétaires ». Cet article qui clôt notre dossier est à la fois un écho aux interrogations soulevées par les autres articles sur la question essentielle et urgente de l'insertion des populations en difficulté, et en même temps une mise en perspective angoissante qui rend dérisoire cette problématique lorsque l'on considère le devenir de ces populations des bidonvilles « abandonnées à l'intégrisme religieux, seule alternative aux mafias ». Même effet d'écho et de dérision sur la question patrimoniale de "la ville officielle » qui se patrimonialise, se cherche artificiellement des racines, se met en scène pour la consommation, les loisirs, la sociabilité, "le plaisir de ses classes moyennes aisées» qui se caractérise par «le souci permanent d'être de toutes les expériences d'en multiplier les sensations mais toujours dans l'éphémère et la dispersion ». Fructueuse et terrible constatation qui relativise les questions de la ville qui nous est familière, en l'ouvrant à la dimension mondiale par ses aspects les plus criants et cependant les plus secrets, ceux de la " planète Bidonville». 